\title{
An Automatic Vibroscope and How to Use It
}

\author{
By Kazuo Wakayama and Eiichi Jinen, Members, TMSJ
}

\author{
Faculty of Textile Fibers, Kyoto University \\ of Industrial Arts and Textile Fibers, Kyoto
}

\begin{abstract}
This article describes an automatic vibroscope of a compact type simpler than the one perfected by Mackay in 1958. Its features include a vibrator to set a fiber in motion, a simple photo-electric transducer to detect fiber vibrations, a direct coupled amplifier of detected electrical signals and a method to adjust the signal phase.

The article shows how to build and operate the new apparatus, and refers to the happy combination of direct coupled amplifier and the pickup-transducer of fiber vibrations. It will be found that this apparatus also does the work of the so-called vibroscope.

Experiments to measure fiber deniers were made under the usual operating conditions with the use of this apparatus, and the sensitivity and accuracy of measuring will probably give a new appraisal to the automatic vibroscope.

Some of the experiments made by the author with the new apparatus may, it is hoped, solve problems, not approached hitherto, on the use of the vibroscopic method.
\end{abstract}

\section{Introduction}

Under proper conditions for measurement, the vibroscopic method measures, with a high degree of sensitivity, the mass per unit length of fibers, filaments and yarns. The measurement is based on the observation of quantities apparently unrelated to the properties of fibers. The method can be applied simply and rapidly even to individual single fibers only several centimeters long without damaging the samples for subsequent tests.

In practice, vibroscopes generally used measure the frequency of excitation or the tension with which a fiber vibrates at resonance. Reading the values of the frequency or the tension is nothing difficult,since it is an ordinary task during observation, but finding the resonance is a task which includes a number of problems calling for a solution. Vibroscopes now in use must be replaced with simple, automatic ones if the vibroscopic method is to do more work than is generally expected of it.

The common procedure of operating vibroscopes, now in use[1], is to place the fiber in a constant length and adjust manually the frequency of excitation or the tension in the fiber, depending on whether the vibroscope used is a constant tension type (TRI type)[2] or a constant frequency type (AV type)[3]. The operation must be continued until the fundamental resonance evidenced by a maximum in the amplitude of fiber vibration is obtained.

The precision with which the frequency or the tension can be adjusted for the maximum amplitude, therefore, depends on the "sharpness of tuning" of the fiber vibrating system and the sensitivity of measuring the amplitude. However the atmosphere dulls the sharpness of tuning so much that the detection of tuning is subject to some personal error [4].

Except in determining the variance of denier in samples of the same kind, it very often happens that fibers of a totally unknown denier is excited at resonance. We must know first of all, then, whether the resonant frequency is that of the fundamental vibration or the higher mode. However, since vibroscopes use a microscope as a detector for us to see the vibration mode as well as the amplitude, it is a troublesome job to move the detector along the fiber. The more sensitive is the detector, the more troublesome is the operation. After the vibration mode was seen, the maximum in the amplitude of fiber vibration must be ascertained with the detector again while the frequency or the tension is being adjusted continuously by hand. This manual operation on several handred samples would be an unbearable job[5]. 
Moreover, detecting a maximum in amplitude is essencially inconsistent with the theory to go by. If a perfect flexible string of the length $l$ (gauge length) and the uniform mass per unit length $\sigma$ is subjected to tension $T$, it may resonate to an excitation at its natural overtone frequency $f_{n}$ of transverse vibrations, given by

$$
f_{n}=n \cdot f_{\vartheta}=n \frac{1}{2 l} \sqrt{T / \sigma_{0}} ; \quad n=1,2, \cdots \quad \cdots \cdots(1)
$$

or $\quad \sigma_{0}=T / 4 l^{2}\left(f_{n} / n\right)^{2}$,

where $f_{\theta}$ is the fundamental frequency.

The equation assumes, however, that the transverse displacement $y$ of a point $x$ on the string at time $t$ is governed by the equation

$$
\sigma\left(\partial^{2} y / \partial t^{2}\right)=T\left(\partial^{2} y / \partial x^{2}\right) ; y=0 \text { at } x=0 \text { or } l \cdots \cdots
$$

where the equation is applicable only to the vibration of a small amplitude.

If the amplitude of fiber vibrations increases, the approximation will lose its validity for deriving the right-hand side of eq. (2) which means the restoring force. At the same time, the tension applied to the string varies slightly from the assumed value $T$; this is a point which Stuart discussed[5].

The automatic vibroscope method of determining the counts of individual fiber may obviate all the above-mentioned difficulties associated with vibroscopes now in use. The only observation necessary in the automatic vibroscope method is to read the frequency at which the fiber is vibrating by selfexcitation. Many other advantages of the method have already been described by Mackay and Downes[7], who, as far as we know, developed the automatic vibroscope at the first time. This article describes a simpler automatic vibroscope than theirs.

Operational functions of our new apparatus are sufficient for various needs. It does also exactly the same work which the manually adjusted vibroscope does. Half of this article is devoted to experiments which used the new apparatus, named "DENIRSCOPE" for brevity, and deals with some problems, not approached hitherto, on the vibroscopic measurement of fiber denier. The results of these experiments may help in assessing the accuracy and sensitivity of measurement with our new apparatus under the usual operating conditions.

\section{Make-up of the New Apparatus}

The automatic vibroscope differs essentially from the manually adjusted vibroscope, in that a fiber is not excited into mechanical resonance by an external source but is regarded as a self-excited mechanical vibrator. The basic task of the automatic vibroscope is to keep the circulation of the signal of fiber vibration in a self-oscillatory loop[7]. The loop is formed, in practice, by linking the transducer for driving fibers (vibrator), the fiber, the device for sensing fiber vibrations (pickup or detector), and the electrical amplifier in a circle. The signal of fiber vibrations, therefore, is transformed properly between the electrical form and the mechanical form in the course of circulation. The method of positive feedback must be used at any point in the loop.

Under nearly sufficient conditions, such a system of the mechano-electric oscillatory loop can oscillate spontaneously with a rather large amplitude in the frequency near the natural frequency of the fiber. Thereafter, the amplifier gain can be reduced gradually by hand so long as the oscillation continues, though with slight variations in the frequency.

At this time, the amplifier seems to adjust itself so as to shift the frequency of the above spontaneous oscillation to the natural frequency of the fiber, and make the most of the amplitude gain in fiber vibrations which is maximum at its natural frequency. At the final stage of this gain reduction, therefore, the frequency of the spontaneous oscillation or the self-oscillation can be read as the natural frequency of the fiber.

Fig. 1 shows the make-up of the new apparatus. The oscillatory loop is a circulating path connecting

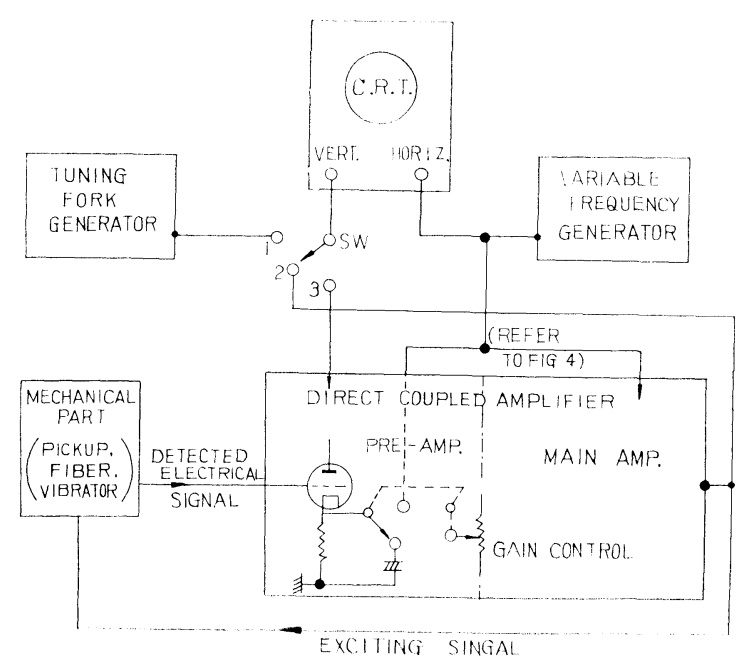

Fig. 1 Make-up of the apparatus 
the electrical amplifier and the mechanical part which includes the vibrator, the fiber and the detector. To see the frequency of the loop oscillation, compare its signal with the electric output from the calibrated audio-frequency generator on the Lissajous pattern on the cathod ray tube.

With the rotary switch SW, in the figure, occasionally placed on the contact point 1 , the output frequency of the audio-frequency generator can be calibrated by that of the standard tuning-fork generator. The frequency of the self-excitedly vibrating fiber can be easily measured to an accuracy of 1 cycle per second.

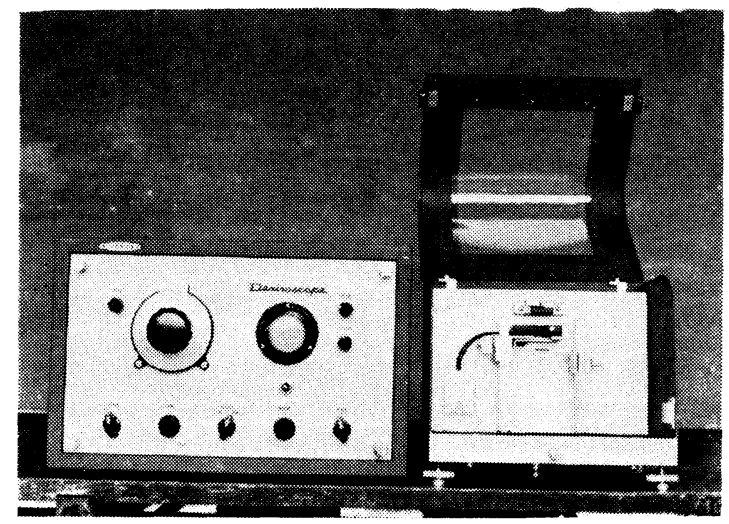

Fig. 2 New Automatic Vibroscope (Deniroscope)

Fig. 2 is a photograph showing our automatic vibroscope. The mechanical part is separated from the electrical part. The latter consists of the audio-frequency generator, the tuning-fork generator, the stabilized power source and the cathod ray tube used for many purposes of measurement. Special experiments, therefore, can be made by the mechanical part under special conditions.

\section{Mechanical Part}

The stereograph in Fig. 3 shows the main section of the mechanical part. The fiber clamped by the clip passes over the upper fret sticking out of the vibrator and is held over the lower fret by the fiber tension a weight attached to the fiber produces. The position of the lower fret can be varied to limit the gauge length from the fiber, to the extent of $12 \mathrm{~cm}$. The movement of the lower fret takes place in the vertical line passing the upper fret. The fiber of any gauge length, therefore, contacts

with the lower fret forming the constant angle, when the mechanical part as a whole is inclined by some angle $\left(2^{\circ}\right)$ from its levelled position.

It is clear from the foregoing descriptions that our new apparatus is an automarized version of vibroscopes of the constant tension type in common use. To use this apparatus for a fiber of a totally unknown denier, a gauge length and a tension must be properly selected by estimate, referring to the errors induced by the usual vibroscopic measurement. Four weights are provided stepwise for this purpose, covering a range of measurement to about 100 denier in fiber fineness.

Shown in the center of Fig. 3 is the photo-electric transducer to detect a vibrating fiber electrically. Its operation will be described in detail in a later section. The transducer is movable vertically along the stand bar. It can, therefore, be fixed in the position where light beams come close to the middle of the gauge length of a fiber. Furthermore, the transducer has to be so movable in the direction perpendicular to the vibrating plane of the fiber, or parallel to the optical axis of light beams, that the fiber is adjustable to the position just in front of the slit.

Choosing a vibrator to drive fibers was one of the difficult problems in our experiments. Since, for many reasons, the electrostatic device [2] was not used, an electro-mechanical transducer would have had to be used in such a way as to vibrate one end

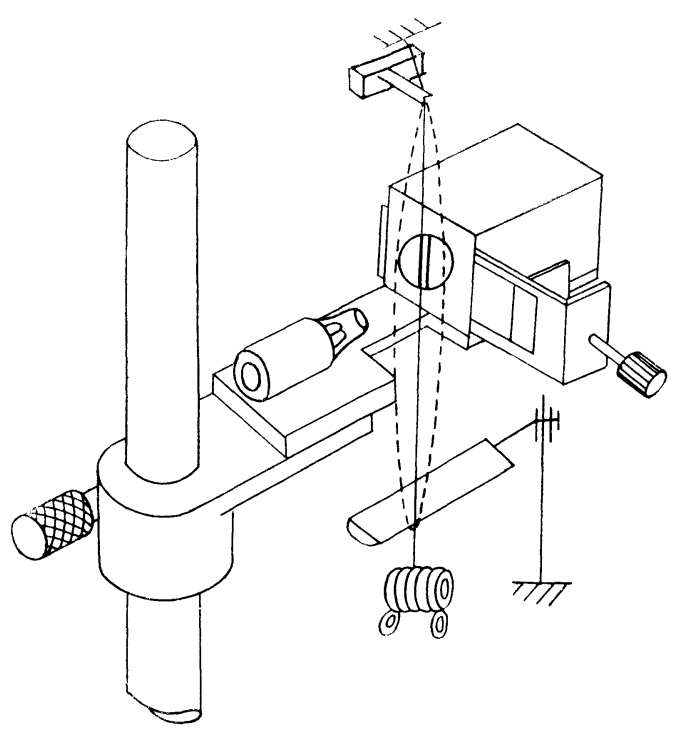

Fig. 3 Principal parts of the mechanical part

Journal of The Textile Machinery Society of Japan 
of the fiber restrained with two frets. The type of transducers, however, would have been accompanied by various correlated difficulties, such as the mechanical output of the vibrator, the natural frequency of the vibrator itself and its mechanical dimension. Besides, another consideration demanded that shorter fibers can be vibrated.

As an alternative, a piezo-electric element of small dimension was used as the vibrator. It bends transversely by electric inputs. By this means we were able to vibrate fibers under a gauge length as short as $25 \mathrm{~mm}$ at a frequency of about $3.5 \mathrm{kc}$.

\section{Amplifier}

One of the requirements imposed on any system in steady-state oscillation is that the total gain and the total phase shift of a signal around an oscillatory loop always are 1 and 0, respectively. Each element in the loop, of course, appropriately contributes some gain and some phase shift, which ordinarily vary with the frequency of the signal. The frequency-dependent characteristic of each loop element, therefore, had to be considered in order to satisfy the conditions for oscillating the loop at various frequencies.

If a fiber fixed at one end is vibrated sinusoidally at the other end, the fiber resonates to excitation at its natural frequency with a particular phase difference and a particular amplitude gain from the vibration of the vibrator. The phase shift, in particular, can be estimated as a constant value independent of the frequencies at which fibers resonate.

Therefore, provided the elements of the loop, except the fiber, are designed so that the algebraic sum of phase shifts in them is constant, provided the sum is nearly cancelled by the phase shift caused by the resonating fiber, and provided the amplifier has a fairely larger gain, the fiber will control itself to vibrate spontaneously.

Naturally, the phase shift and the amplitude gain of the excited fiber vary rapidly with frequency in the vicinity of resonance. Therefore, if the selfoscillation frequency slightly shifts from the natural frequency of the fiber due to overloading in the amplifier, the conditions for self-oscillation will be satisfied enough.

The foregoing considerations suggest that a direct coupled amplifier doubtless is suitable for an electrical amplifier of loop elements. The phase shift previously fixed in direct coupled amplifiers naturally shows no variation with frequencies of the signal. Furthermore, we shall see later that the direct coupled amplifier is indispensable for the generation and stability of oscillation in the loop in conjunction with the functions of the slit.

The direct coupled amplifier we found satisfactory for use was of a rather simple type and was supplied with a direct-current source of $300 \mathrm{~V}$. All its stages were connected by a neon tube and direct couplings.

When the width of the slit is varied according to the fineness of sample fibers and the main switch is turned on, the bias voltage has to be adjusted in the direct coupled amplifier. A cathod ray tube is handy for that purpose and makes it possible to

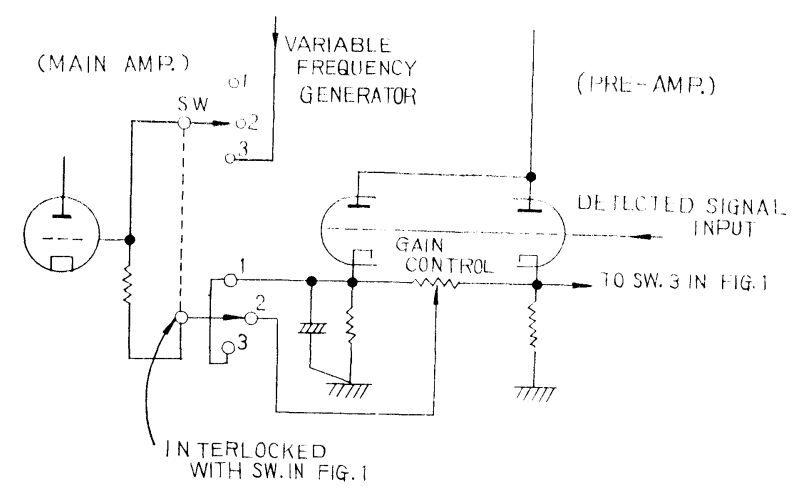

Fig. 4 Circuit diagram showing the device for gain control and electrical division of the D-C amplifier

adjust the amplifier manually while visually making sure that the amplifier is kept in full action. The electric circuit (Fig. 1) is adjusted by inserting a small signal into the first stage of the amplifier from the audio-frequency generator.

The direct coupled amplifier may be expected to be useful, as we have already said, but generally, its use is accompanied by a few difficulties. The drift is one of them, but it can be excluded from consideration here. For observation with automatic vibroscopes usually takes only a short time, and the amplifier does not need much gain in amplification.

However, the method of gain control certainly presents a difficulty. Changes in the bias-voltage and in the phase relation of the amplifier should not accompany the control of gain. The electric circuit shown in Fig. 4 completely solves the difficulty. The selection switch is interlocked with the switch in Fig. 1 and usual measurements are made in the state of contact point 2 of the switch. 
The push-pull output from the last stage of the amplifier drives the piezo-electric element. The output source is so low in impeadance as to prevent the amplifier from oscillating electrically due to some coupling in the electric system.

The vibrator, which itself necessarily exhibits mechanical resonance at some frequencies, has a minimum resonant frequency of about $5 \mathrm{kc}$. The mechanical natural vibrations of the vibrator were not particularly damped, because the damping effects make variations in phase shift with frequency larger. Therefore, the gauge length and the weight must be selected under the condition which limits the selfoscillatory frequencies of fibers to about $3.5 \mathrm{kc}$.

\section{Functions of the Slit}

The pickup shown in Fig. 3 consists of a lenseformed nipple bulb, a slit, and a photo-transistor. It can shift horizontally at a small angle around the stand bar. This shifting is operated smooth by manually rotating the knop in front of the mechanical body. The faint shadow of the fiber, therefore, traverses perpendicularly the edges of the slit parallel to the fiber. The other conditions being satisfied, the fiber will vibrate instantaneously as the shadow falls on either edges of the slit.

The positive phase shift is given to the oscillatory signal on this edge of the slit, although the other elements in the loop usually produce negative phase shifts. The total sum of the phase shifts, therefore, can equal 0 around the loop. The direct action of the positive phase shifting is as follows:

The falling of the shadow on the edge produces a decrease in the light beam. The vibrator can then be expected to be bent so nicely by the output amplified through the photo-transistor from the decrease as to drive out the fiber from the slit. Remember that this operation is possible only by the use of the direct coupled amplifier, because the phenomena are not rapid enough for alternatingcurrent amplifiers to work sufficiently.

Then, the light beam which enters the phototransistor is increased by the emergence of the shadow from the slit, and the fiber tends to return to the first position which was brought manually. The fiber, therefore, continues to vibrate. On the contrary, the action which takes place as the shadow falls on the other edge of the slit does not help to induce oscillation, because of the negative feedback.
It seems very difficult to put a filament as fine as 1 denier on the edge of the slit, as we have already said. Nevertheless, it was not necessary to prepare a special fine screw to shift the pickup in front of the slit. Besides, the oscillation in the loop was so stable that fiber vibrations of $0.02 \mathrm{~mm}$ in amplitude could be maintained after the gain of amplifier was reduced. Moreover, when a long filament was pulled over two smooth frets at a constant speed and tension, the fiber vibrations were stable as long as a somewhat larger amplitude was allowed.

Here are the explanations of these facts. A vibrator driven by an alternating-current amplifier has only a neutral position on its supports. Therefore, the edge of the slit cannot be expected to square-except by chance-with the fiber hanging over the vibrator. In contrast, the direct coupled amplifier allows the vibrator to take a neutral position arbitrarily to some extent. This means that the fiber apparently appears thicker than it is. Therefore, the edge can more easily keep the shadow of the fiber in line.

The degree within which the fiber is nearly parallel to the edge of the slit essentially has a bearing on the efficiency of photo-electric transformation in the pickup. Yet rule-of-thumb adjustment of parallelism suffices. The direct coupled amplifier is unsuited to the triangular slit used by Rabbidge and Leary[8]. We can see, then, what good the combination of the direct coupled amplifier and the pickup is intended to give the full functions. Also the fundamental mode of fiber vibrations is always obtainable.

\section{Adjustment of Phase Shift}

We have said that a constant phase difference exists between the vibrations of the vibrator and those of the fiber at resonance, no matter what the frequency is. The requirement for phase shift in the loop elements, except the fiber and the vibrator, has given birth to the pickup and the direct coupled amplifier for our apparatus. The last loop element, the vibrator, has a disadvantage: Slight variations in phase shift with frequencies are unavoidable owing to a small electric capacitance in the vibrator. The variations were compensated for by the phase shifter of an integration type at the first stage in the amplifier. The shifter can vary the phase to $10^{\circ}$ at the frequency of $3.5 \mathrm{kc}$. 
To make the phase shifter do its work fully, throw the interlocking rotary switch SW onto contact point 3 (Fig. 4). This divides the amplifier electrically into two unrelated parts between two stages in it. The main part of the amplifier, serving as the output amplifier of the variable audio-frequency generator, drives the fiber. The pre-amplifier serves as the detecting amplifier of the vibrating fiber excited by the above main amplifier. At the same time, outputs from the main amplifier and the detecting amplifier both are inserted, respectively, into $\mathrm{X}$ axis and $\mathrm{Y}$ axis of the cathod ray tube, forming Lissajous patterns on the surface of the tube.

The above switching has the effect of only cutting the oscillatory loop in two at the place shown in Figs. 1 and 4. The static-electrical state of the amplifier remains unchanged. Therefore, if the self-oscillatory frequency of the fiber is copied on the variable audio-frequency generator under the state of the circuit switched on contact point 2, and if the circuit is then switched over to contact point 3 , then the Lissajous pattern on the tube will show a straight line which makes some angle to the horizontal (Fig. 5b). This shows conclusively that the frequency satisfies the condition of self-oscillation of the loop which has the fiber as a element.

With the frequency of the generator slightly raised or lowered, the Lissajous pattern varies as shown in Figs. 5a to $5 \mathrm{c}$, displaying long ellipses. The reason is that the phase in the signal varies so rapidly near the natural frequency of the fiber. A visually detectable variation in the Lissajous pattern is detected by our apparatus as a result of the frequency variation of about 0.5 c.p.s.

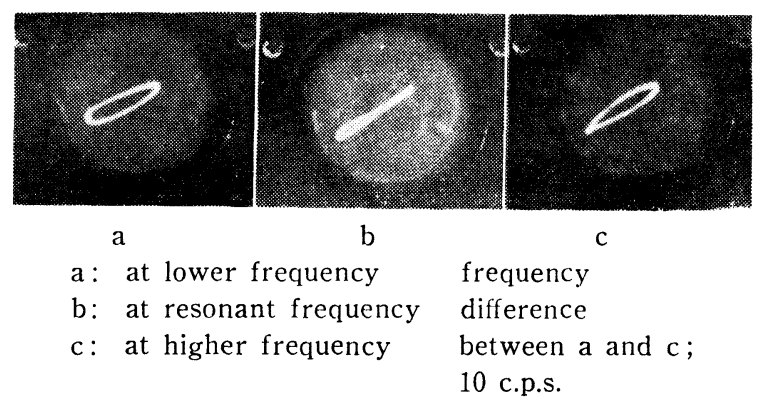

Fig. 5 Changes in Lissajous figure shown by the signals of excitation and fiber vibrations

The amplitude of fiber vibrations always appears as Y-deflections on the cathod ray tube. Taking a different viewpoint, we may say that the above manual operation is exactly the same as that of so- called vibroscopes of the constant tension type. In this apparatus a cathod ray tube is used instead of a microscope.

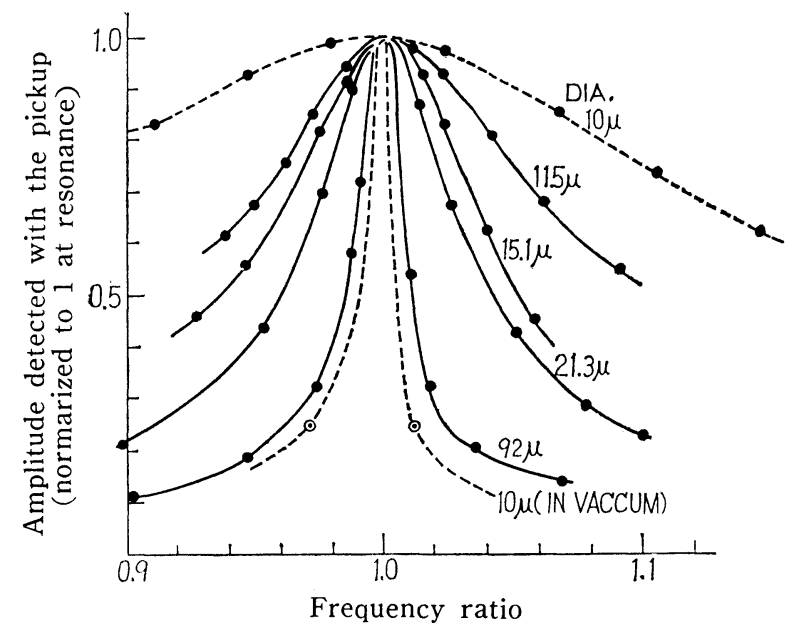

Fig. 6 Resonance curves detected with the pick-up for forced vibrating fibers (Various diameter) in air, the broken line showing measurements with a telescope cited from the Stauff's paper[4]

Fig. 6 shows examples of fiber resonance curves. The ordinate of the figure indicates the relative values of the amplitude measured on the cathod ray tub for signals picked up from fiber vibrations. This curve differs from a result obtained by Stauff and Montgomery [4] in the sharpness of tuning. This point is being investigated.

How to adjust the phase will be clear from the functions of our apparatus. The phase shifter must be so adjusted in the last-mentioned state of the amplifier that the Lissajous pattern makes a straight line at the frequency whose excitation vibrates the fiber in maximum amplitude.

The phase relation is fixed fairly properly in the amplifier. The natural frequency of the fiber, therefore, doubtless exists near that of the loop oscillation which anyways takes place, irrespective of the phase shifter, in the circuit state of contact point 2 of the switch. The phase shifter, then, should be thought as a fine adjuster not needed for rough measurements.

\section{Accuracy and Sensitivity}

Every quantity on the right-hand side of eq. (1) is easily measurable to an accuracy of $0.1 \%$. It is extremely easy to fix frequencies down to 1 c.p.s., so that we can keenly observe even variations in resonant frequency resulting from the regain of the 
fiber which has been breathed upon. Denier values simply calculated from such measurements, however, still need some corrections according to the conditions accompanying casual observations.

Table 1 Systematic Errors Induced by Vibroscopic Measurement of Fiber Linear Density $\sigma$ Using the Vibroscope of Constant-tension Type

\begin{tabular}{lcc}
\multicolumn{1}{c}{ Source of error } & \multicolumn{2}{c}{ Signs of errors } \\
& in frequency & $\begin{array}{c}\text { in } \sigma \text { calculated } \\
\text { from the Eq.(1) }\end{array}$ \\
\hline 1. Surrounding air & - & + \\
2. Non-uniformity & indefinite & - (error in) \\
3. Extension & + & - \\
4. Form of cross section & indefinite & - (error in) \\
5. Stiffness & + & - \\
6. Friction on the fret & - & + \\
7. Amplitude of vibrations & + & - \\
8. Motion of support & - & +
\end{tabular}

Table 1 shows the sources of corrections and the signs of errors caused by such conditions. Note that $f$ is an observed value and $\sigma$ is a value obtained by substituting $f$ in eq. (1). Most of the data in this table have been tabulated by Mackay[7] and studies by many researchers[4,6,9 11]. Their reports had better be consulted by users of the vibrating method. Items which directly concern our new apparatus are No. 7, No. 8, and part of No. 6 .

The amplitude of fiber vibrations can be reduced to a value as small as $0.02 \mathrm{~mm}$, depending on how the amplifier gain is controlled. The upper fret supports the fiber rigidly enough, as we shall see from the fact that the transverse vibrations of the fiber are driven by the longitudinal motion of the fret which is made of a piece of thin metal. We may assume, then, that the correction of Nos. 7 and 8 in the usual measurements with our apparatus is negligible.

In the measuring system of the vibrating method shown in Fig. 7, the angle $\theta$ must be so fixed that the tension in the fiber is large enough to hold the fiber securely to the fret. In this instrument, even an angle as small as $2^{\circ}$ suffices, because the amplitude of fiber vibrations is very small. Therefore, it is also possible to regard the gravity of a weight as the tension in the fiber. With the angle as small as $2^{\circ}$, the effect of the fiber-fret friction can often be ignored.

We notice several unsolved problems about the vibrating method of determining the fiber linear density. They will be experimentally dealt with in the next three sections, where a new appraisal will be made of the accuracy, sensibility and simplicity of measurement with our new apparatus under usual operating conditions.

\section{Friction at the Lower Fret}

The lower fret is movable along the vertical stand bar and limits the arbitrary length of the fiber to the gauge length, as shown in Fig. 7. The friction given to the fiber on the fret, therefore, has a different effect, depending on whether the fret is placed in a given position through an upward or downward displacement. This means that the fiber obtains different tensions $T$ from that assumed by a weight $M$ in the part of the fiber limited to the gauge length.

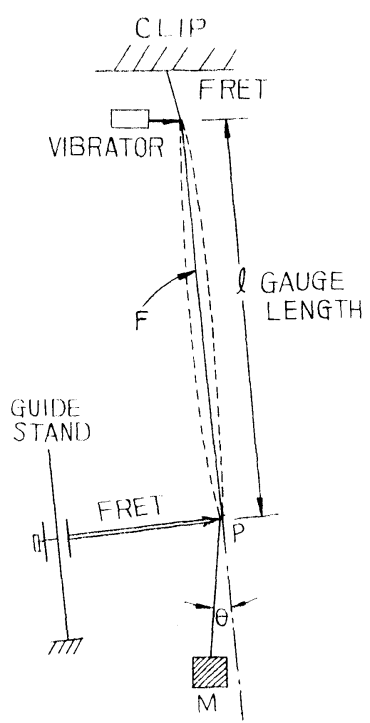

Fig. 7 Setting up of the fiber in the instrument

From Stuart's discussions[6] we can quote the formula :

$$
M \cdot g \cdot e^{-\mu \theta}<T<M \cdot g \cdot e^{\mu \theta},
$$

where $\mu$ is the coefficient of friction between the fret and the sample fiber. The formula results in $3 \mu \theta / 4$, as the width $\Delta f / f$ of variations in the natural frequency calculated from eq. (1). The selfoscillatory frequency was experimentally determined by the two ways of placing the fret in a given position, and a mean value $\Delta f=29$ c.p.s. at $\bar{f}=1388$ c.p.s. was obtained as a result. Calculation from 
this result gives $\mu=0.79$.

This experiment suggests a method of placing the sample fiber on our apparatus. After the fiber has hung over the frets, it should be held with a strip of paper or something at the point $F$, and shaken about. This treatment subjects the part of the fiber limited to the gauge length nearly to the minimum tension in the range indicated by eq. (3), i.e., $T=M g e^{-\mu \theta}$

\section{Effects of the Gauge Length}

The effect of stiffness is usually prominent among the effects of the various sources of errors, and is especially so in measurements made with our apparatus. The fact can be understood from these experiments: The fiber under a constant tension was allowed to vibrate in different gauge lengths $l$ at their respective self-oscillatory frequency $f$. In the right of eq. (1), frequency $f$ was plotted as the function of $1 / l$, as shown in Fig. 8 . The curve appearing in a linear relation apparently does not pass the origin. Correction of the result, therefore, can perhaps be estimated from the effect of stiffness for fiber oscillation.

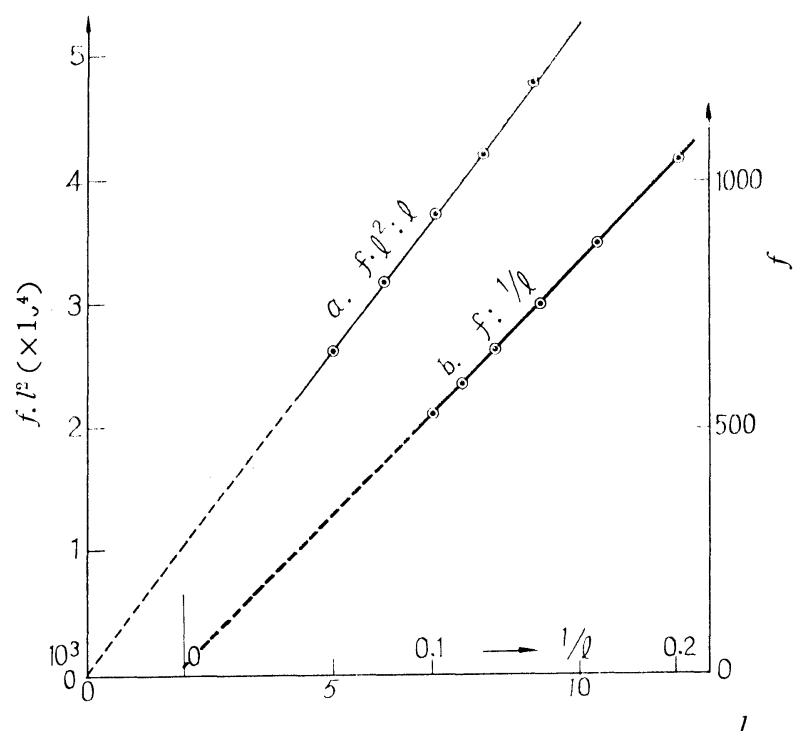

Fig. 8 Relations between the gauge length $(l)$ and frequency $(f)$ under vibroscopic measurement of fiber counts

Assuming that the fiber has Young's modulus $E$ and moment of inertia $I$ concerning a neutral axis, the theory of the transverse vibrating string induces the following equation as an approximation to the first order in the fundamental oscillation:

$$
\left.\begin{array}{l}
f=f_{\theta}(1+\alpha) \\
\sigma_{0}=\sigma(1+2 \alpha)
\end{array}\right\} ; \quad \sigma=\frac{T}{4 l^{2} f^{2}}, \quad \alpha=\sqrt{\frac{4 E I}{l^{2} T}}
$$

We have already said that $f$ is an observed value and $\sigma$ is a value calculated from the simple equation. Quantities accompanied by suffixes express their respective unknown true values.

Using eq. (1) in eq. (4) gives us:

$$
\begin{aligned}
f & =\left(f_{\theta} \cdot l^{2}+f_{0} \cdot l^{2} \alpha\right) / l^{2} \\
& =\left(\sqrt{T / \sigma_{0}} \cdot l / 2+\sqrt{\left.E T / \sigma_{0}\right)}\right) / l^{2}
\end{aligned}
$$

A curve which verifies eq. (6) is shown in Fig. 8 , too, indicating a straight line. It is believed that the segment which the curve, as a straight line, and the abscissa sever from the ordinate expresses the second term in eq. (6). Numerical examinations of the term as well as $\alpha$ in eq. (4), then, can be given as follows:

Assuming $I=S^{2} / 4 \pi$ for the fiber having a circular section whose area is $S$, and assuming $\rho$ as the density of the fiber, then:

the second term $=\sqrt{\sigma_{0} E / \pi} / 2^{\circ}$,

and

$$
\alpha=\sigma_{0} \sqrt{E / \pi T} / l_{\rho}
$$

With $\sigma$ and $T$ expressed by denier $d$ and the weight of mass $M(g)$,

the secorid term $=\sqrt{E d_{0} / \rho} / 3.36 \times 10^{3}$

and

$$
\alpha=d_{0} \sqrt{E / M} / 4.99 \times 10^{7} \cdot l \cdot \rho
$$

Assuming that $E=5 \times 10^{10} \mathrm{dyn} / \mathrm{cm}^{2}$ and $\rho=1.2$ $\mathrm{g} / \mathrm{cm}^{3}$ for most textile fibers, then :

the second term $=55 \sqrt{d_{0}}$

and

$$
\alpha=0.0037 d_{0} / l \sqrt{M}
$$

The value of the second term for the observed fiber is about 120, which agrees well with the length of the segment. These and other experiments go to prove that attention only to stiffness nearly suffices for correcting the values simply calculated by eq. (1) so long as our apparatus is used.

In practice, $\alpha$ in eqs. (7), (8), and (9) must be looked up before choosing values for use as $l$ and $M$. In contrast, $\alpha$ must be estimated, after observation, from the following approximate equation which uses observed values, instead of $f_{0}$ and $d_{0}$ :

$$
f \cdot l^{2} \cdot \alpha=55 \sqrt{d}
$$

which is obtained from comparing the two second terms in eqs. (5) and (6).

The longer the gauge length, the smaller the value of the correction factor favorably. Remember, 
however, that the damping effect of the air around the fiber becomes noticeable if the gauge length is too long. Therefore, the correction factor had better not be used as a value below $0.5 \%$ when $l$ and $M$ are selected (Table 2).

Table 2 Self-oscillatory Frequencies of Filament Vibrating to Various Gauge Lengths and Corrections by Stiffness to the Vibroscopic Denier (Sample: Tetoron, $M=0.7 \mathrm{~g}, d=5.2$ )

\begin{tabular}{cccc}
\hline $\begin{array}{c}\text { Gauge length } \\
l(\mathrm{~cm})\end{array}$ & $\begin{array}{c}\text { Frequency } \\
f \text { (c.p.s.) }\end{array}$ & $f \cdot l^{2}$ & $\begin{array}{c}\text { Correction } \\
\text { for stiffness } \\
2 \alpha(\%)\end{array}$ \\
\hline 5 & 1041 & 26025 & 0.92 \\
6 & 882 & 31752 & 0.76 \\
7 & 762 & 37338 & 0.64 \\
8 & 658 & 42112 & 0.58 \\
9 & 592 & 47952 & 0.50 \\
10 & 526 & 52600 & 0.46 \\
\hline
\end{tabular}

\section{Effect of the Form in Fiber Cross Section}

Montgomery and Milloways [2] derived an expression of the correction factor in which the form in the cross section was considered:

$$
\alpha=\sqrt{\eta} \sqrt{\frac{E S^{2}}{\pi l^{2} T}}
$$

The quantity $\eta$ takes different values depending on the form in the fiber cross section and equals 1 for the circular section. Some uncertainty, therefore, surrounds the value of $\eta$, because, in practice, it is not possible to put a finger on the plane of vibration in the fiber.

The only experiment possible to prove this fact will require statistical processing of data. This experiment is to clamp the same sample to the apparatus over and over again. The statistical denier data, then, are made of the measurements on various plane of vibration in the fiber. Fig. 9 is some examples of these experiments. The distribution of observed deniers is slightly out of normal for a case where fibers are used as a sample, but deniers of the metal wire which was drawn through a die make a tendency to the normal distribution.

\section{Continuous Tests of Yarn Evenness}

The fiber placed in our automatic vibroscope
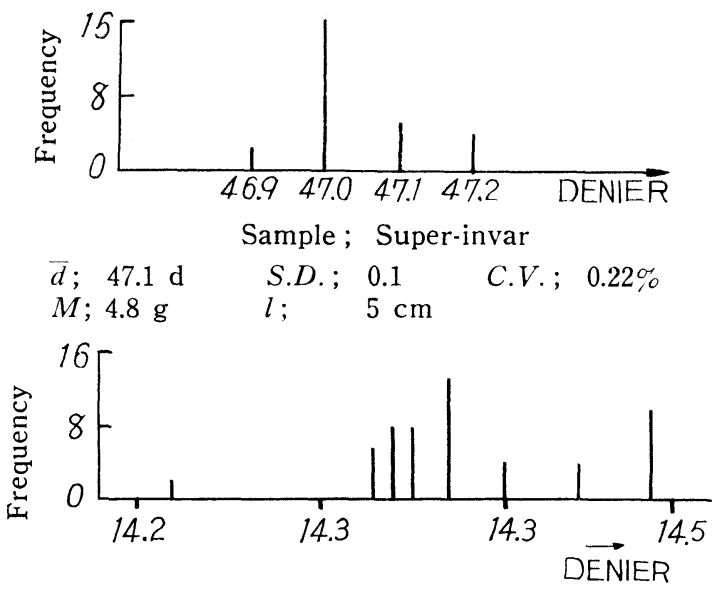

$\begin{array}{llll}d ; 14.4 \mathrm{~d} & \text { S.D. } ; & 0.061 & \text { C.V. } ; 0.43 \% \\ M ; 1.2 \mathrm{~g} & l ; & 4 \mathrm{~cm} & \end{array}$

Fig. 9 Frequency distribution of repeated denier measurement on same sample clamped over and over again to the apparatus
$d:$ Mean of deniers
$M:$ Weight
S.D. : Standard deviation $l:$ Gauge length
C.V. : Coefficient of variation

vibrates instantaneously and the vibrations continue stably. We, therefore, thought of using this features in our continuous tests of yarn unevenness [12]. In the light of eq. (1), the following relation is derivable under a constant $l$ and $T$ :

$$
\frac{\Delta \sigma}{\sigma}=2 \frac{\Delta f}{f}
$$

A yarn is driven over the two frets in front of the pickup of the automatic vibroscope, and the variations $\Delta f$ in self-oscillatory frequency $f$ show on recording paper. Then we can see variations in yarn evenness in percentage proportional to $\Delta f$.

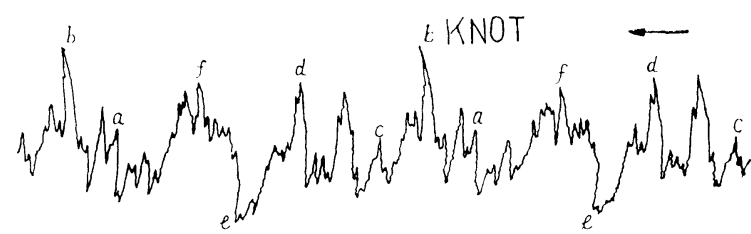

Fig. 10 Recordings of continuous tests of evenness by the vibrating method with the new apparatus (Cotton yarn 30's; $2 \mathrm{~m}$ loop endlessly showing 2 cycles in circulation of the loop)

Fig. 10 is an example of recordings obtained by using the sensitive descriminator of frequency variations. It is designed especially for this purpose. The sample was a cotton yarn $2 \mathrm{~m}$ long. It was connected endlessly to make a loop, and was driven 
over the mechanical part of our new automatic vibroscope at a speed of about $5 \mathrm{~m} / \mathrm{min}$. Two cycles in circulation of the looped yarn can be easily read in the figure, where the point marked $b$ means the knot on the looped yarn.

Fig. 11 shows a similar result obtained from the same sample with USTER evenness tester. This curve is compared with the another recording which was obtained from vibrating the same yarn pulled at a different speed. The marks $a, b$, - on the recording correspond to those in Fig. 10.

Conformity of the two kinds of recordings in Fig. 11 to each other is nearly complete as to a large period along the length. During these experiments, the sample yarn happened to be so thick that USTER tester could be used. Therefore, the results of the experiments seem to verify the usefulness of USTER evenness tester. Remember, however, that the vibrating string method is indispensable for thin fibers, for which USTER tester is useless.

\section{Various Types of Uniformity in Filaments}

Uniformity or regularity in filaments has been variously discussed by many researchers. This section deals with uniformity experiments of various meanings and compares them with one another.

In Fig. 12, the regularity in denier along the length is expressed in the form of a frequency distribution. Data on the copper wire, which was drawn through dies, were obtained from measurements of 20 places, $7 \mathrm{~cm}$ apart, along the length. They show the normal distribution. In contrast to the metal wire, the deniers of nylon filament are distributed with slight normality. The range of the distribution clearly exceeds that caused by the uncertainty in fiber section which has been foresaid in section 10 (Fig. 9). These deniers were measured at 40 places, $9 \mathrm{~m}$ apart, along the length.

Multi-filament yarns make the distributions shown in Fig. 13, where all the filaments in each yarn were vibrated. The coefficients of variance calculated from these measurements are fairly greater than the variance coefficients from the measurements along the length of long filaments (Fig. 12). Table 3 will interest users of the vibrating string method for denier measurement. The mean denier calculated from measurements on every filament in yarns is compared with the denier measured by vibrating a whole, undivided multi-filament yarn and by weighing it whole.

The samples used in the above experiments were stretched under a tensile tester, and the respective

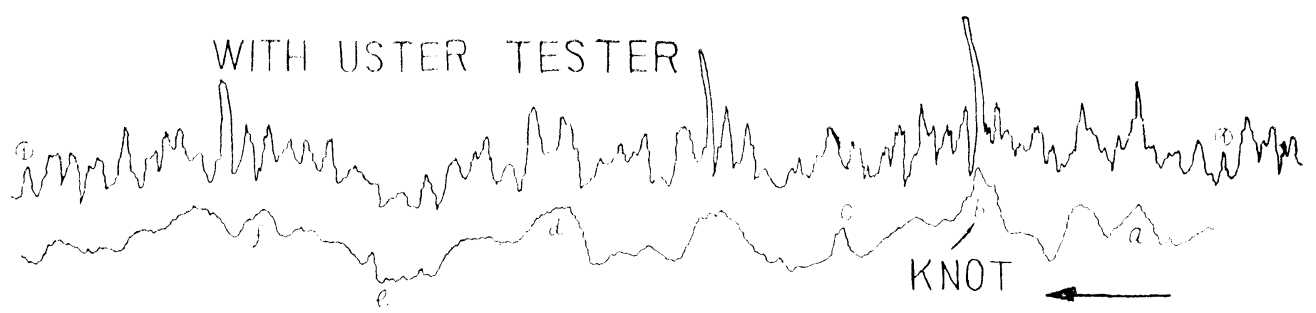

By the vibrating method

Fig. 11 Comparison of recordings by two methods for the continuous tests of evenness with Uster tester and the new automatic vibroscope

(Cotton yarn 30's; the same sample with Fig. 10; Marks corresponding to that in Fig. 10)

Table 3 Mean Deniers of Multi-filament Yarns Measured by Different Methods.

$d$ : Mean of deniers; $M$ : Weight $(\mathrm{g})$; Correction factor $(\%)$

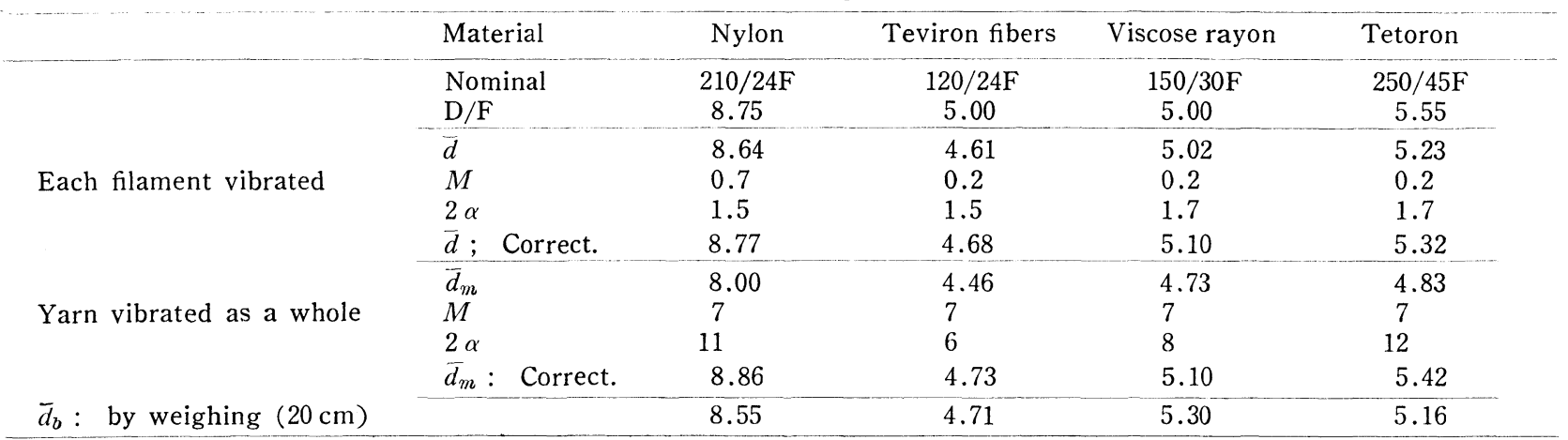


breaking strengths of the individual filaments were measured. To estimate the mean breaking stress, one of two methods is used, depending on whether the denier of each filament is known or unknown. If it is known, the breaking stress of each filament is calculated and averaged. If the denier is unknown, the mean of breaking strengths must necessarily be divided by the nominal denier of the multi-filament yarn. Table 4 shows a comparision of estimates of breaking stress made by the two methods, although

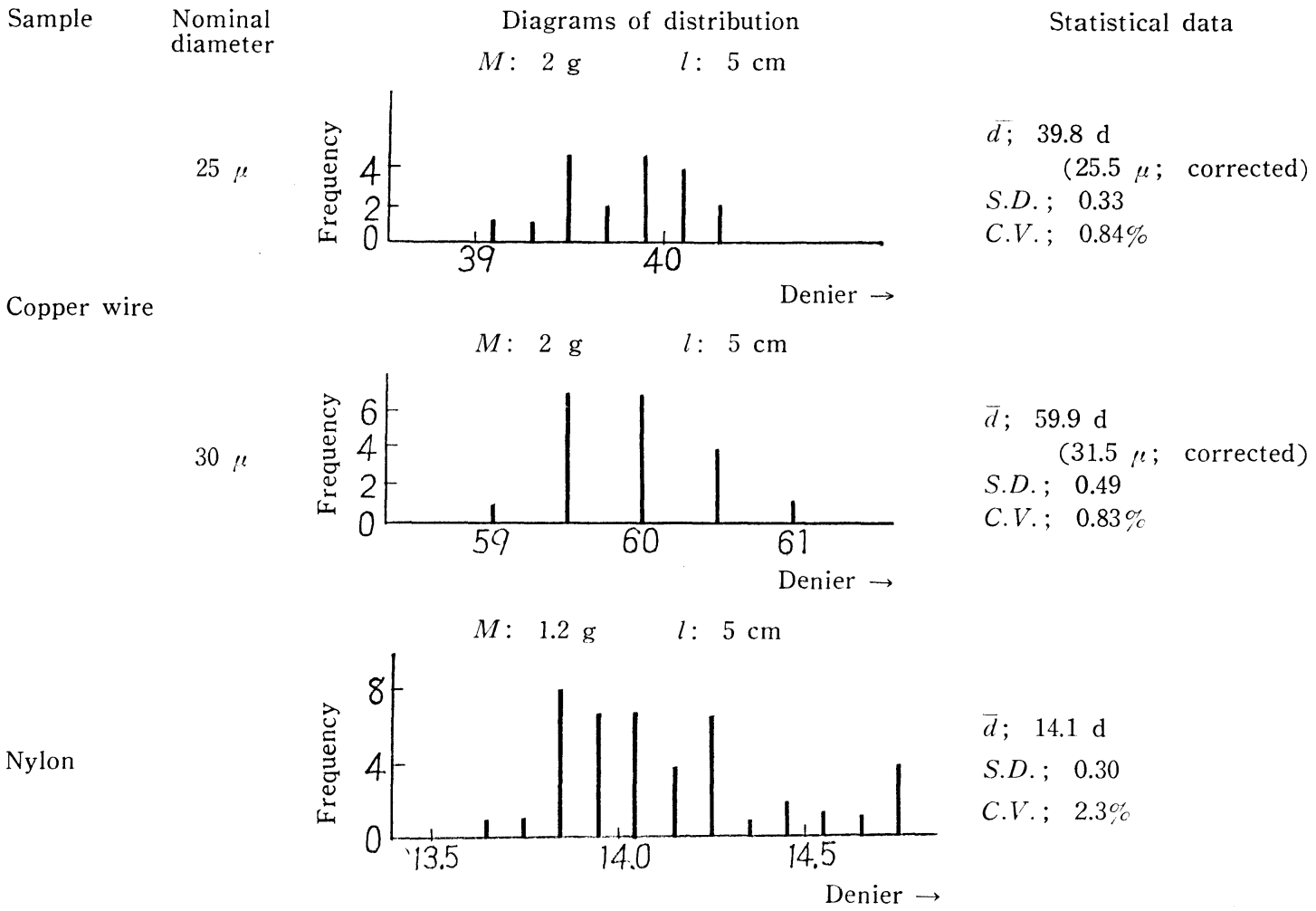

Fig. 12 Uniformity in denier along length of filaments

$\bar{d}:$ Mean of deniers

$M$ : Weight
S.D. : Standard deviation

$l$ : Gauge length
$C . V .:$ Coefficient of variance

C.V.: Coefficient of variance

Table 4 Comparison of Calculations of Breaking Stress by Two Methods

$\bar{g}$ : Mean of breaking stress; $\quad \bar{d}:$ Mean of deniers

S.D. : Standard deviation C.V.: Coefficient of variance (\%)

\begin{tabular}{lcccc}
\hline Material & Nylon & Teviron & $\begin{array}{c}\text { Viscose } \\
\text { rayon }\end{array}$ & Tetoron \\
Nominal & $210 \mathrm{D} / 24 \mathrm{~F}$ & $120 \mathrm{D} / 24 \mathrm{~F}$ & $150 \mathrm{D} / 30 \mathrm{~F}$ & $250 \mathrm{D} / 45 \mathrm{~F}$ \\
$\overline{g / d}$ & 7.12 & 3.11 & 1.97 & 6.91 \\
S.D. & 0.60 & 0.45 & 0.18 & 0.80 \\
C.V. & 8.5 & 14.5 & 9.3 & 11.6 \\
g/d & 7.10 & 3.04 & 1.73 & 6.93 \\
S.D. & 0.67 & 16.5 & 16.22 & 0.84 \\
C.V. & 9.4 & 16.9 & 12.7 & 12.1
\end{tabular}


Sample

Nylon

$210 \mathrm{D} / 24 \mathrm{~F}$

$M: \quad 0.7 \mathrm{~g}$

$l: 5 \mathrm{~cm}$
Teviron $\quad M: 0.2 \mathrm{~g}$

$120 \mathrm{D} / 24 \mathrm{~F}$

$l: 5 \mathrm{~cm}$
Tetoron

$250 \mathrm{D} / 45 \mathrm{~F}$
M: $0.2 \mathrm{~g}$

$l: 5 \mathrm{~cm}$
Diagrams of distribution
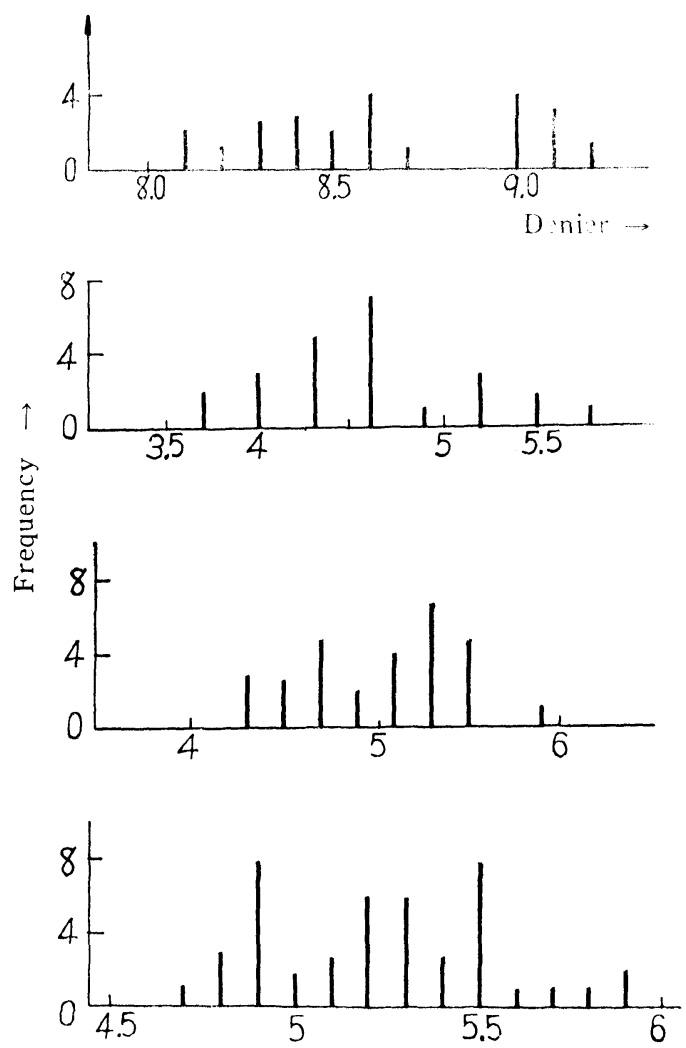

Statistical data

$\bar{d} ; 8.64 \mathrm{~d}$

S.D. ; 0.36

C.V. ; $4.2 \%$

$d ; 4.61 \mathrm{~d}$

S.D. ; 0.6

C.V. ; $13 \%$

d $5.02 \mathrm{~d}$

S.D. ; 0.41

C.V. ; $8.1 \%$

$\bar{d} ; 5.23 \mathrm{~d}$

S.D. ; 0.31

C.V. ; $5.9 \%$

Fig. 13 Uniformity in denier of multi-filament yarns, all filaments in each yarn, vibrated in the new apparatus

$\bar{d}:$ Mean of deniers

$M:$ Weight
S.D. : Standard deviation

$l$ : Gauge length the mean denier was used instead of the norminal. In the table $g / d$ means a mean value of $g / d$ computed with each denier known; $g / d$, a value computed with each denier unknown.

\section{Summing Up}

It is common knowledge that an automatic vibroscope is useful as a new version of the vibroscopes now frequently used to measure the linear density of fibers, filaments and yarns. There are, however, a number of technical difficulties attending the use of an automatic vibroscope. They concern methods of exciting fibers, detecting fiber vibrations and adjusting the phase shift around an oscillatory loop. Because of these difficulties, hardly any instrument of a simpler type, except the one perfected by Mackay, has been around.

Our study which is reported on this article has overcome the difficulties by using a piezo-electric element of very small dimensions, a photo-transistor, a special device for detecting fiber vibrations, a direct coupled amplifier and a special device for dividing the amplifier in two electrically. As a result, a compact apparatus has been developed as a unit instrument. Its compactness is shown by the fact that it uses only one cathod ray tube for many different types of operations.

The advantages of our instrument as an automatic vibroscope cannot be over-emphasized. It practically excludes the personal error associated with the detection of resonant vibration, gives con- 
tinuous indication and is very rapid in response. It is the special advantage that the instrument can display the rapid changes in phase variation which the fiber vibration shows nearby the resonant point.

The latter half of this article helps to estimate the last mentioned as well as the precision and sensitivity of measument with the new apparatus and solves the problems, not hitherto approached, on the use of the vibroscopic method.

Some experiments concerned the effect of the friction between the fiber and the fret over which the fiber hangs, and of selection of the gauge length and the weight to be applied to the fiber. Various information has been obtained also from the effect which the form in the fiber cross section has on the measurement of denier and from a comparison of various types of fibers uniformity and their magnitude. Moreover, continuous tests of yarn evenness with our automatic vibroscope showed that its record agrees completely with results obtained from USTER evenness tester. The fact has been unexpected.

This study could not have been made without the financial assistance of TOYO RAYON CO., LTD. and the Science and Technology Agency of the Japanese Ministry of International Trade and Industry, to whom we express our gratitude.

The authors thank Dr. T. Narita, Dr. C. Oh-oka, Dr. A. Mukoyama, Dr. E. Fujimoto, Prof. T. Hattori and Prof. S. Oh-ya for their constant interest and encouragement. Thanks are due also to Dr. V. D. Burgmann of the C.S.I.R.O. Wool Research Laboratory for his generous supply of valuable publications on this project.

\section{Literature}

[1] A.S.T.M. Designation D 1577-58T

[2] D.T. Montgomery, W.T. Milloway; Text. Res. J., 22, 729 (1952)

[3] S.L. Dart, L.E. Peterson; Text. Res. J., 19, 38 (1949)

[4] D.W. Stauff, D.T. Montgomery: J. Appl. Phys., 26, 540 (1955)

[5] H. Wakeham, W.P. Virgin, N. Spicer; Text. Res. J., 24, 802 (1954)

D.H. Morton; J. Text. Inst., 47, T422 (1956)

[6] I.M. Stuart; Brit. J. Appl. Phys., 10, 219 (1959)

[7] B.H. Mackay, J.G. Downes; Text. Res. J., 28, 467 (1958)

[8] R.M. Rabbidge; C.S.I.R.O., Report No. S21, (1960)

[9] E.T.L. Voong, D.T. Montgomery; Text. Res. J., 23, 821 (1953)

[10] D.J. Montgomery; J. Appl. Phys., 24, 1092 (1953)

[11] D.H. Patt; Text. Res. J., 28, 691 (1958)

[12] J.G. Downes, B.G. Leary; Text. Res. J., 28, 497 (1958) R.A. Sack; Brit. J. Appl. Phys., 5, 224 (1954)

B-G. Leary; Instrument Practice and Electronics, 12, 1179 (1958) 